\title{
A "SUPERRESOLUTION" TARGET-TRACKING CONCEPT
}

\author{
William F. Gabriel \\ Electromagnetics Branch \\ Radar Division \\ Naval Research Laboratory \\ Washington, D.C. 20375
}

\begin{abstract}
"Superresolution" techniques are an emerging technology which presents opportunteies for achieving significant improvements in the detection/tracking of closely-spaced multiple sources and targets. The new technology derives largely from modern spectral estimation theory/technlques of the past decade, and adaptive array antenna processing systems $[1,2,3]$. Such systems are termed "data adaptive" because they adapt to the total signal environment "seen" by the array of antenna elements, using all available degrees-offreedom of the system in an optimal least-mean-square estimation error sense.
\end{abstract}

The particular system concept to be described in this paper has been simplified to the block diagram shown in Fig. 1. Note that it represents an all-digital processing system, commencing with the A/D (analog-to-digital) converters at the antenna elements (or beam ports if it is a beamformer type of antenna). The system continuously computes/updates a sample covariance matrix, $\underline{R}$, averaged over $\mathrm{N}$ snapshots (range cells),

$$
\underline{R}=\frac{1}{N} \sum_{n=1}^{N} E_{n}^{*} E_{n}^{t}
$$

where $E_{n}$ is a column vector containing the nth snapshot element signal samples.

Off-line processing upon $\underline{R}$ is then conducted at pertodic intervals to estimate the locations and relative power levels of interfering sources. There are literally dozens of algorithms available in the spectral estimation literature for making such estimates; ranglng from fast simple routines [1,2] for estlmating strong sources, to the more sophisticated Eigen-analysis routines $[2,3]$ for estimating weak sources. Once the locations of the sources are determined, one can solve for the relative source power levels to assist in screening out "false alarms" [2]. The computed source information is then utilized to periodically update digital spatial filter optimized adaptive weights which filter out all sources that are logically decermined to be interference.

The filtered signal residue is next processed by search/track beamformer algorithms to sift out and track the targets of interest.

To demonstrate the concept, Fig. 2 illustrates a simple simulation example in which we have a single $3 \mathrm{~dB}$ SNR (signal-tonoise ratio) target located at $-4 \mathrm{deg}$. azimuth and appearing in a

U.S. Government work not protected by U.S. copyright. 
single range bin per PRF (pulse repetition frequency) period. There are also six interference sources of relative strengths and locations as noted, which appear in $\mathrm{N}$ adjacent range bins per PRF period. The two $10 \mathrm{~dB}$ SNR sources at -42 and -54 deg. are $95 \%$ coherent. An 8 element linear array is the receiving aperture, with elements spaced half-wavelength. Upon forming sample covariance matrices $\mathrm{R}_{f}$ averaged over 256 range bin samples, and processing with an Eigenanalysis algorithm, we get a digital output estimation plot of relative source power level vs, azimuth angle location as 1llustrated in Fig. 3 for 4 successive estimation trials. Note that the estimates in Fig. 3 are reasonably close to the truth given in Fig. 2, with the results being best for the strong sources, as one might expect. The estimated source information is then applied to compute digital filter optimized adaptive weights which filter out the interference sources as illustrated by Fig. 4, wherein is plotted the spatial filter "insertion loss" vs. azimuth angle. Fig. 4 illustrates three important points: (a) we apply only enough adaptive insertion loss to drop an interference source into the noise; (b) the insertion loss "cost" against a desired target will increase as the target gets closer to a strong interference; and (c) if the target SNR is sufficient to overcome the insertion loss, we can track within a beamwidth of a strong interference, i.e., achieve "superresolution". The filtered signal residue in this example permitted easy monopulse tracking of the desired target pulses contained in the residue, as illustrated in Fig. 5 .

A caveat regarding such optimized adaptive filtering concepts is that they usually involve non-1inear processing of the signals, and one may anticipate wavefront distortion problems which can cause tracking errors.

1. W.F. Gabrie1, "Spectral Analysis and Adaptive Array

Superresolution Techniques", Proc. IEEE, Vol. 68, June 1980.

2. First ASSP Workshop on Spectral Estimation, McMaster

University, Hamilton, Ontario, August 1981.

3. Special Issue on Spectral Estimation, Proc. IEEE, Vol. 70, September 1982 . 


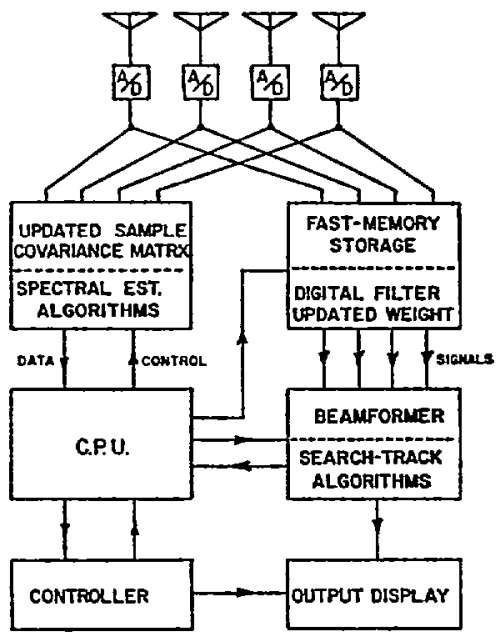

Fig. 1 - Adaptive Array Tracking System Concept

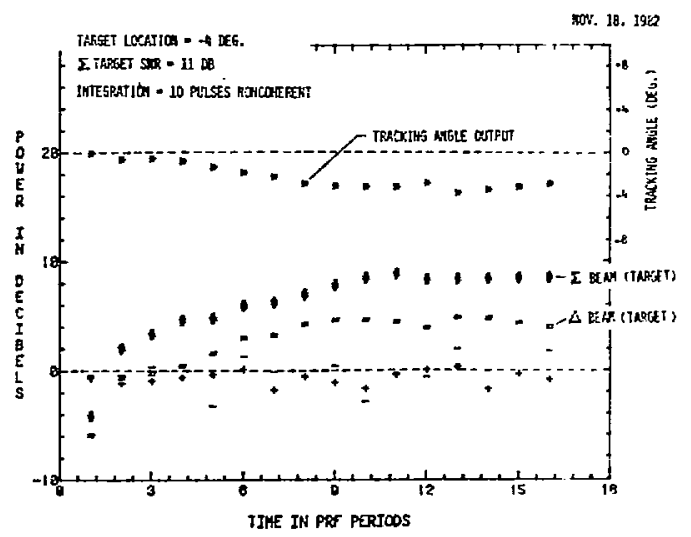

Fig. 5 - Monopulse Tracking Beam Outputs 

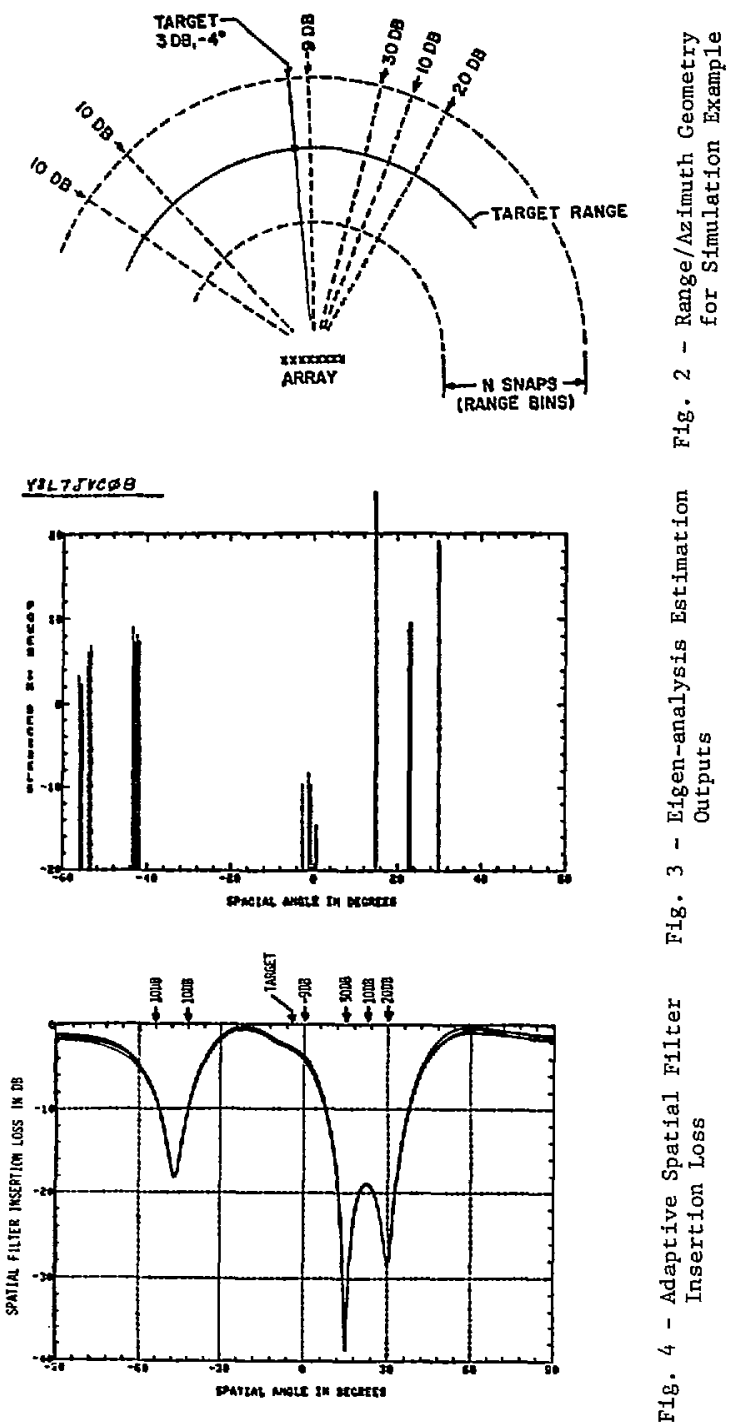